\title{
On Fractional Integral Inequalities Involving Hypergeometric Operators
}

\author{
D. Baleanu, ${ }^{1,2,3}$ S. D. Purohit, ${ }^{4}$ and Praveen Agarwal $^{5}$ \\ ${ }^{1}$ Department of Mathematics and Computer Sciences, Faculty of Arts and Sciences, Cankaya University, 06530 Ankara, Turkey \\ ${ }^{2}$ Department of Chemical and Materials Engineering, Faculty of Engineering, King Abdulaziz University, P.O. Box 80204, \\ Jeddah 21589, Saudi Arabia \\ ${ }^{3}$ Institute of Space Sciences, Magurele Bucharest, Romania \\ ${ }^{4}$ Department of Basic Sciences (Mathematics), College of Technology and Engineering, M.P. University of Agriculture and Technology, \\ Udaipur 313001, India \\ ${ }^{5}$ Department of Mathematics \& Statistics, J.E.C.R.C. University, Jaipur 303905, India
}

Correspondence should be addressed to S. D. Purohit; sunil_a_purohit@yahoo.com

Received 21 September 2013; Accepted 4 November 2013; Published 15 January 2014

Academic Editors: C. Yin and H. You

Copyright (C) 2014 D. Baleanu et al. This is an open access article distributed under the Creative Commons Attribution License, which permits unrestricted use, distribution, and reproduction in any medium, provided the original work is properly cited.

Here we aim at establishing certain new fractional integral inequalities involving the Gauss hypergeometric function for synchronous functions which are related to the Chebyshev functional. Several special cases as fractional integral inequalities involving Saigo, Erdélyi-Kober, and Riemann-Liouville type fractional integral operators are presented in the concluding section. Further, we also consider their relevance with other related known results.

\section{Introduction}

Fractional integral inequalities have many applications; the most useful ones are in establishing uniqueness of solutions in fractional boundary value problems and in fractional partial differential equations. Further, they also provide upper and lower bounds to the solutions of the above equations. These considerations have led various researchers in the field of integral inequalities to explore certain extensions and generalizations by involving fractional calculus operators. One may, for instance, refer to such type of works in the book [1] and the papers [2-11].

In a recent paper, Purohit and Raina [9] investigated certain Chebyshev type [12] integral inequalities involving the Saigo fractional integral operators and also established the $q$-extensions of the main results. The aim of this paper is to establish certain generalized integral inequalities for synchronous functions that are related to the Chebyshev functional using the fractional hypergeometric operator, introduced by Curiel and Galué [13]. Results due to Purohit and Raina [9] and Belarbi and Dahmani [2] follow as special cases of our results.

In the sequel, we use the following definitions and related details.

Definition 1. Two functions $f$ and $g$ are said to be synchronous on $[a, b]$, if

$$
\{(f(x)-f(y))(g(x)-g(y))\} \geq 0
$$

for any $x, y \in[a, b]$.

Definition 2. A real-valued function $f(t)(t>0)$ is said to be in the space $\mathbb{C}_{\mu}(\mu \in \mathbb{R})$, if there exists a real number $p>\mu$ such that $f(t)=t^{p} \phi(t)$, where $\phi(t) \in \mathbb{C}(0, \infty)$.

Definition 3. Let $\alpha>0, \mu>-1$, and $\beta, \eta \in \mathbb{R}$; then a generalized fractional integral $I_{t}^{\alpha, \beta, \eta, \mu}$ (in terms of the Gauss 
hypergeometric function) of order $\alpha$ for a real-valued continuous function $f(t)$ is defined by [13] (see also [14]):

$$
\begin{aligned}
I_{t}^{\alpha, \beta, \eta, \mu}\{f(t)\}= & \frac{t^{-\alpha-\beta-2 \mu}}{\Gamma(\alpha)} \\
& \times \int_{0}^{t} \tau^{\mu}(t-\tau)^{\alpha-1} \\
& \times{ }_{2} F_{1}\left(\alpha+\beta+\mu,-\eta ; \alpha ; 1-\frac{\tau}{t}\right) f(\tau) d \tau,
\end{aligned}
$$

where the function ${ }_{2} F_{1}(-)$ appearing as a kernel for the operator (2) is the Gaussian hypergeometric function defined by

$$
{ }_{2} F_{1}(a, b ; c ; t)=\sum_{n=0}^{\infty} \frac{(a)_{n}(b)_{n}}{(c)_{n}} \frac{t^{n}}{n !}
$$

and $(a)_{n}$ is the Pochhammer symbol:

$$
(a)_{n}=a(a+1) \cdots(a+n-1), \quad(a)_{0}=1 .
$$

The object of the present investigation is to obtain certain Chebyshev type integral inequalities involving the generalized fractional integral operators [13] which involves in the kernel, the Gauss hypergeometric function (defined above). The concluding section gives some special cases of the main results.

\section{Main Results}

Our results in this section are based on the preliminary assertions giving composition formula of fractional integral (2) with a power function.

Lemma 4. Let $\alpha, \beta, \eta, \lambda \in \mathbb{R}, \mu>-1, \mu+\lambda>0$, and $\lambda-\beta+$ $\eta>0$, then the following image formula for the power function under the operator (2) holds true:

$$
I_{t}^{\alpha, \beta, \eta, \mu}\left\{t^{\lambda-1}\right\}=\frac{\Gamma(\mu+\lambda) \Gamma(\lambda-\beta+\eta)}{\Gamma(\lambda-\beta) \Gamma(\lambda+\mu+\alpha+\eta)} t^{\lambda-\beta-\mu-1} .
$$

Proof. To prove (5), we take $f(\tau)=\tau^{\lambda-1}$ in the definition of fractional integral operator $I_{t}^{\alpha, \beta, \eta, \mu}(\cdot)$, given by (2), the lefthand side (say $\mathscr{L}$ ) yields to

$$
\begin{aligned}
\mathscr{L}= & \frac{t^{-\alpha-\beta-2 \mu}}{\Gamma(\alpha)} \\
& \times \int_{0}^{t} \tau^{\mu+\lambda-1}(t-\tau)^{\alpha-1}{ }_{2} F_{1}\left(\alpha+\beta+\mu,-\eta ; \alpha ; 1-\frac{\tau}{t}\right) d \tau .
\end{aligned}
$$

Now, on using the following integral formula involving the Gaussian hypergeometric function [15, page 106, equation (3.118)]:

$$
\begin{aligned}
\int_{0}^{t} \tau^{\rho-1}(t-\tau)^{c-1}{ }_{2} F_{1}\left(a, b ; c ; 1-\frac{\tau}{t}\right) d \tau \\
=\frac{\Gamma(c) \Gamma(\rho) \Gamma(\rho+c-a-b)}{\Gamma(\rho+c-a) \Gamma(\rho+c-b)} \tau^{\rho+c-1},
\end{aligned}
$$

then (6) immediately leads to the result (5).

Now, we obtain certain integral inequalities for the synchronous functions involving the generalized fractional integral operator (2).

Theorem 5. Let $f$ and $g$ be two synchronous functions on $[0, \infty)$; then

$$
\begin{aligned}
I_{t}^{\alpha, \beta, \eta, \mu} & \{f(t) g(t)\} \\
\geq & \frac{\Gamma(1-\beta) \Gamma(1+\mu+\alpha+\eta) t^{\beta+\mu}}{\Gamma(1+\mu) \Gamma(1-\beta+\eta)} \\
& \times I_{t}^{\alpha, \beta, \eta, \mu}\{f(t)\} I_{t}^{\alpha, \beta, \eta, \mu}\{g(t)\},
\end{aligned}
$$

for all $t>0, \alpha>\max \{0,-\beta-\mu\}, \beta<1, \mu>-1, \beta-1<\eta<0$.

Proof. Let $f$ and $g$ be two synchronous functions; then using Definition 1 , for all $\tau, \rho \in(0, t), t \geq 0$, we have

$$
\{(f(\tau)-f(\rho))(g(\tau)-g(\rho))\} \geq 0,
$$

which implies that

$$
f(\tau) g(\tau)+f(\rho) g(\rho) \geq f(\tau) g(\rho)+f(\rho) g(\tau) .
$$

\section{Consider}

$$
\begin{aligned}
F(t, \tau) & \\
= & \frac{t^{-\alpha-\beta-2 \mu} \tau^{\mu}(t-\tau)^{\alpha-1}}{\Gamma(\alpha)} \\
& \times{ }_{2} F_{1}\left(\alpha+\beta+\mu,-\eta ; \alpha ; 1-\frac{\tau}{t}\right) \\
= & \frac{\tau^{\mu}}{\Gamma(\alpha)} \frac{(t-\tau)^{\alpha-1}}{t^{\alpha+\beta+2 \mu}}+\frac{\tau^{\mu}(\alpha+\beta+\mu)(-\eta)}{\Gamma(\alpha+1)} \frac{(t-\tau)^{\alpha}}{t^{\alpha+\beta+2 \mu+1}} \\
& +\frac{\tau^{\mu}(\alpha+\beta+\mu)(\alpha+\beta+\mu+1)(-\eta)(-\eta+1)}{\Gamma(\alpha+2)} \\
& \times \frac{(t-\tau)^{\alpha+1}}{t^{\alpha+\beta+2 \mu+2}}+\cdots, \quad(\tau \in(0, t) ; t>0) .
\end{aligned}
$$

We observe that each term of the above series is positive in view of the conditions stated with Theorem 5 , and hence the function $F(t, \tau)$ remains positive, for all $\tau \in(0, t)(t>0)$.

Multiplying both sides of (10) by $F(t, \tau)$ (where $F(t, \tau)$ is given by (11)) and integrating with respect to $\tau$ from 0 to $t$, and using (2), we get

$$
\begin{aligned}
I_{t}^{\alpha, \beta, \eta, \mu} & \{f(t) g(t)\}+f(\rho) g(\rho) I_{t}^{\alpha, \beta, \eta, \mu}\{1\} \\
& \geq g(\rho) I_{t}^{\alpha, \beta, \eta, \mu}\{f(t)\}+f(\rho) I_{t}^{\alpha, \beta, \eta, \mu}\{g(t)\} .
\end{aligned}
$$

Next, multiplying both sides of (12) by $F(t, \rho)(\rho \in(0, t), t>$ 0 ), where $F(t, \rho)$ is given by (11), and integrating with respect 
to $\rho$ from 0 to $t$, and using formula (5) (for $\lambda=1$ ), we arrive at the desired result (8).

The following results give a generalization of Theorem 5 .

Theorem 6. Let $f$ and $g$ be two synchronous functions on $[0, \infty)$ then

$$
\begin{aligned}
& \frac{\Gamma(1+\mu) \Gamma(1-\beta+\eta)}{\Gamma(1-\beta) \Gamma(1+\mu+\alpha+\eta) t^{\beta+\mu}} I_{t}^{\gamma, \delta, \zeta, v}\{f(t) g(t)\} \\
& \quad+\frac{\Gamma(1+\nu) \Gamma(1-\delta+\zeta)}{\Gamma(1-\delta) \Gamma(1+\nu+\gamma+\zeta) t^{\delta+\nu}} I_{t}^{\alpha, \beta, \eta, \mu}\{f(t) g(t)\} \\
& \geq I_{t}^{\alpha, \beta, \eta, \mu}\{f(t)\} I_{t}^{\gamma, \delta, \zeta, v}\{g(t)\} \\
& \quad+I_{t}^{\gamma, \delta, \zeta, v}\{f(t)\} I_{t}^{\alpha, \beta, \eta, \mu}\{g(t)\},
\end{aligned}
$$

for allt $>0, \alpha>\max \{0,-\beta-\mu\}, \gamma>\max \{0,-\delta-\nu\}, \mu, \nu>-1$, $\beta, \delta<1, \beta-1<\eta<0, \delta-1<\zeta<0$.

Proof. To prove the above theorem, we use inequality (12). Multiplying both sides of (12) by

$$
\begin{array}{r}
\frac{t^{-\gamma-\delta-2 v} \rho^{\nu}(t-\rho)^{\gamma-1}}{\Gamma(\gamma)}{ }_{2} F_{1}\left(\gamma+\delta+\nu,-\zeta ; \gamma ; 1-\frac{\rho}{t}\right) p(\rho) \\
(\rho \in(0, t) ; t>0),
\end{array}
$$

which remains positive in view of the conditions stated with (13) and integrating with respect to $\rho$ from 0 to $t$, we get

$$
\begin{aligned}
& I_{t}^{\gamma, \delta, \zeta, \nu}\{1\} I_{t}^{\alpha, \beta, \eta, \mu}\{f(t) g(t)\}+I_{t}^{\alpha, \beta, \eta, \mu}\{1\} I_{t}^{\gamma, \delta, \zeta, \nu}\{f(t) g(t)\} \\
& \geq I_{t}^{\alpha, \beta, \eta, \mu}\{f(t)\} I_{t}^{\gamma, \delta, \zeta, \nu}\{g(t)\} \\
& \quad+I_{t}^{\gamma, \delta, \zeta, \nu}\{f(t)\} I_{t}^{\alpha, \beta, \eta, \mu}\{g(t)\},
\end{aligned}
$$

which on using (5) readily yields the desired result (13).

Remark 7. It may be noted that inequalities (8) and (13) are reversed if the functions are asynchronous on $[0, \infty)$; that is,

$$
\{(f(x)-f(y))(g(x)-g(y))\} \leq 0,
$$

for any $x, y \in[0, \infty)$.

Remark 8. For $\gamma=\alpha, \delta=\beta, \zeta=\eta$, and $v=\mu$, Theorem 6 immediately reduces to Theorem 5 .

Theorem 9. Let $\left(f_{i}\right)_{i=1, \ldots, n}$ be n positive increasing functions on $[0, \infty)$; then

$$
\begin{aligned}
& I_{t}^{\alpha, \beta, \eta, \mu}\left\{\prod_{i=1}^{n} f_{i}(t)\right\} \\
& \geq\left[\frac{\Gamma(1-\beta) \Gamma(1+\mu+\alpha+\eta) t^{\beta+\mu}}{\Gamma(1+\mu) \Gamma(1-\beta+\eta)}\right]^{n-1} \prod_{i=1}^{n} I_{t}^{\alpha, \beta, \eta, \mu}\left\{f_{i}(t)\right\},
\end{aligned}
$$

for all $t>0, \alpha>\max \{0,-\beta-\mu\}, \mu>-1, \beta<1, \beta-1<\eta<0$.
Proof. We prove this theorem by mathematical induction. Clearly, for $n=1$ in (17), we have

$$
I_{t}^{\alpha, \beta, \eta, \mu}\left\{f_{1}(t)\right\} \geq I_{t}^{\alpha, \beta, \eta, \mu}\left\{f_{1}(t)\right\} \quad(t>0, \alpha>0) .
$$

Next, for $n=2$, in (17), we get

$$
\begin{aligned}
I_{t}^{\alpha, \beta, \eta,} & \left\{f_{1}(t) f_{2}(t)\right\} \\
\geq & \frac{\Gamma(1-\beta) \Gamma(1+\mu+\alpha+\eta) t^{\beta+\mu}}{\Gamma(1+\mu) \Gamma(1-\beta+\eta)} \\
& \times I_{t}^{\alpha, \beta, \eta, \mu}\left\{f_{1}(t)\right\} I_{t}^{\alpha, \beta, \eta, \mu}\left\{f_{2}(t)\right\} \quad(t>0, \alpha>0),
\end{aligned}
$$

which holds in view of (8) of Theorem 5.

By the induction principle, we suppose that the inequality

$$
\begin{aligned}
& I_{t}^{\alpha, \beta, \eta, \mu}\left\{\prod_{i=1}^{n-1} f_{i}(t)\right\} \\
& \quad \geq\left[\frac{\Gamma(1-\beta) \Gamma(1+\mu+\alpha+\eta) t^{\beta+\mu}}{\Gamma(1+\mu) \Gamma(1-\beta+\eta)}\right]^{n-2} \prod_{i=1}^{n-1} I_{t}^{\alpha, \beta, \eta, \mu}\left\{f_{i}(t)\right\},
\end{aligned}
$$

holds true for some positive integer $n \geq 2$.

Now $\left(f_{i}\right)_{i=1, \ldots, n}$ are increasing functions which imply that the function $\prod_{i=1}^{n-1} f_{i}(t)$ is also an increasing function. Therefore, we can apply inequality (8) of Theorem 5 to the functions $\prod_{i=1}^{n-1} f_{i}(t)=g$ and $f_{n}=f$ to get

$$
\begin{aligned}
I_{t}^{\alpha, \beta, \eta, \mu} & \left\{\prod_{i=1}^{n} f_{i}(t)\right\} \\
\geq & \frac{\Gamma(1-\beta) \Gamma(1+\mu+\alpha+\eta) t^{\beta+\mu}}{\Gamma(1+\mu) \Gamma(1-\beta+\eta)} \\
& \times I_{t}^{\alpha, \beta, \eta, \mu}\left\{\prod_{i=1}^{n-1} f_{i}(t)\right\} I_{t}^{\alpha, \beta, \eta, \mu}\left\{f_{n}(t)\right\},
\end{aligned}
$$

provided that $t>0, \alpha>\max \{0,-\beta-\mu\}, \mu>-1, \beta<1$, $\beta-1<\eta<0$.

Making use of (20) now, this last inequality above leads to the result (17), which proves Theorem 9.

Now, we consider another variation of the fractional integral inequalities.

Theorem 10. Let $f$ and $g$ be two functions defined on $[0, \infty)$, such that $f$ is increasing, $g$ is differentiable, and there exists a real number $m=\inf _{t \geq 0} g^{\prime}(t)$; then

$$
\begin{aligned}
I_{t}^{\alpha, \beta, \eta, \mu} & \{f(t) g(t)\} \\
& \geq \frac{\Gamma(1-\beta) \Gamma(1+\mu+\alpha+\eta) t^{\beta+\mu}}{\Gamma(1+\mu) \Gamma(1-\beta+\eta)}
\end{aligned}
$$




$$
\begin{aligned}
& \times I_{t}^{\alpha, \beta, \eta, \mu}\{f(t)\} I_{t}^{\alpha, \beta, \eta, \mu}\{g(t)\} \\
& -\frac{m(1+\mu)(1-\beta+\eta) t}{(1-\beta)(1+\mu+\alpha+\eta)} I_{t}^{\alpha, \beta, \eta, \mu}\{f(t)\} \\
& +m I_{t}^{\alpha, \beta, \eta, \mu}\{t f(t)\},
\end{aligned}
$$

for all $t>0, \alpha>0, \beta<1, \mu>-1, \beta-1<\eta<0$.

Proof. Consider the function $h(t)=g(t)-m t$. It is clear that $h$ is differentiable and it is increasing on $[0, \infty)$; therefore, by using Theorem 5, we get

$$
\begin{aligned}
& I_{t}^{\alpha, \beta, \eta, \mu}\{f(t)(g(t)-m t)\} \\
& \geq \frac{\Gamma(1-\beta) \Gamma(1+\mu+\alpha+\eta) t^{\beta+\mu}}{\Gamma(1+\mu) \Gamma(1-\beta+\eta)} \\
& \times I_{t}^{\alpha, \beta, \eta, \mu}\{f(t)\} I_{t}^{\alpha, \beta, \eta, \mu}\{g(t)-m t\} \\
& \geq \frac{\Gamma(1-\beta) \Gamma(1+\mu+\alpha+\eta) t^{\beta+\mu}}{\Gamma(1+\mu) \Gamma(1-\beta+\eta)} \\
& \times I_{t}^{\alpha, \beta, \eta, \mu}\{f(t)\} I_{t}^{\alpha, \beta, \eta, \mu}\{g(t)\} \\
&-\frac{\Gamma(1-\beta) \Gamma(1+\mu+\alpha+\eta) t^{\beta+\mu}}{\Gamma(1+\mu) \Gamma(1-\beta+\eta)} \\
& \times I_{t}^{\alpha, \beta, \eta, \mu}\{f(t)\} m I_{t}^{\alpha, \beta, \eta, \mu}\{t\} .
\end{aligned}
$$

Now, on making use of formula (5) (for $\lambda=2$ ), we are lead to the result (22) after some simplifications.

Theorem 11. Let $f$ and $g$ be two functions defined on $[0, \infty)$, such that $f$ is increasing, $g$ is differentiable, and there exists a real number $M=\sup _{t \geq 0} g^{\prime}(t)$; then

$$
\begin{aligned}
I_{t}^{\alpha, \beta, \eta, \mu} & \{f(t) g(t)\} \\
\geq & \frac{\Gamma(1-\beta) \Gamma(1+\mu+\alpha+\eta) t^{\beta+\mu}}{\Gamma(1+\mu) \Gamma(1-\beta+\eta)} \\
& \times I_{t}^{\alpha, \beta, \eta, \mu}\{f(t)\} I_{t}^{\alpha, \beta, \eta, \mu}\{g(t)\} \\
& -\frac{M(1+\mu)(1-\beta+\eta) t}{(1-\beta)(1+\mu+\alpha+\eta)} I_{t}^{\alpha, \beta, \eta, \mu}\{f(t)\} \\
& +M I_{t}^{\alpha, \beta, \eta, \mu}\{t f(t)\},
\end{aligned}
$$

for all $t>0, \alpha>0, \beta<1, \mu>-1, \beta-1<\eta<0$.

Proof. By applying the similar procedure as of Theorem 10, one can easily establish the above theorem. Therefore, we omitted the details of the proof of this theorem.

\section{Special Cases}

We now briefly consider some consequences of the results derived in the previous section. Following Curiel and Galué
[13], the operator (2) would reduce immediately to the extensively investigated Saigo, Erdélyi-Kober, and RiemannLiouville type fractional integral operators, respectively, given by the following relationships (see also $[14,16]$ ):

$$
\begin{aligned}
& I_{0, t}^{\alpha, \beta, \eta}\{f(t)\} \\
& \quad=I_{t}^{\alpha, \beta, \eta, 0}\{f(t)\} \\
& \quad=\frac{t^{-\alpha-\beta}}{\Gamma(\alpha)} \int_{0}^{t}(t-\tau)^{\alpha-1}{ }_{2} F_{1}\left(\alpha+\beta,-\eta ; \alpha ; 1-\frac{\tau}{t}\right) f(\tau) d \tau, \quad(\alpha>0, \beta, \eta \in \mathbb{R}), \quad(25) \\
& I^{\alpha, \eta}\{f(t)\} \\
& =I_{t}^{\alpha, 0, \eta, 0}\{f(t)\} \\
& =\frac{t^{-\alpha-\eta}}{\Gamma(\alpha)} \int_{0}^{t}(t-\tau)^{\alpha-1} \tau^{\eta} f(\tau) d \tau \quad(\alpha>0, \eta \in \mathbb{R}), \\
& R^{\alpha}\{f(t)\}=I_{t}^{\alpha,-\alpha, \eta, 0}\{f(t)\} \\
& \quad=\frac{1}{\Gamma(\alpha)} \int_{0}^{t}(t-\tau)^{\alpha-1} f(\tau) d \tau \quad(\alpha>0) .
\end{aligned}
$$$$
(\alpha>0, \beta, \eta \in \mathbb{R})
$$

Now, if we consider $\mu=0$ (and $\nu=0$ additionally for Theorem 6) and make use of relation (25), Theorems 5 to 9 provide, respectively, the known fractional integral inequalities due to Purohit and Raina [9].

Again, for $\mu=0$ Theorems 10 and 11 provide, respectively, the following inequalities involving Saigo fractional integral operators.

Corollary 12. Let $f$ and $g$ be two functions defined on $[0, \infty)$, such that $f$ is increasing, $g$ is differentiable, and there exists a real number $m=\inf _{t \geq 0} g^{\prime}(t)$; then

$$
\begin{aligned}
I_{0, t}^{\alpha, \beta, \eta} & \{f(t) g(t)\} \\
\geq & \frac{\Gamma(1-\beta) \Gamma(1+\alpha+\eta) t^{\beta}}{\Gamma(1-\beta+\eta)} I_{0, t}^{\alpha, \beta, \eta}\{f(t)\} I_{t}^{\alpha, \beta, \eta}\{g(t)\} \\
& \quad-\frac{m(1-\beta+\eta) t}{(1-\beta)(1+\alpha+\eta)} I_{0, t}^{\alpha, \beta, \eta}\{f(t)\}+m I_{0, t}^{\alpha, \beta, \eta}\{t f(t)\}
\end{aligned}
$$

for all $t>0, \alpha>0, \beta<1, \beta-1<\eta<0$.

Corollary 13. Let $f$ and $g$ be two functions defined on $[0, \infty)$, such that $f$ is increasing, $g$ is differentiable, and there exists a real number $M=\sup _{t \geq 0} g^{\prime}(t)$; then

$$
\begin{aligned}
I_{0, t}^{\alpha, \beta, \eta} & \{f(t) g(t)\} \\
& \geq \frac{\Gamma(1-\beta) \Gamma(1+\alpha+\eta) t^{\beta}}{\Gamma(1-\beta+\eta)} I_{0, t}^{\alpha, \beta, \eta}\{f(t)\} I_{0, t}^{\alpha, \beta, \eta}\{g(t)\}
\end{aligned}
$$




$$
-\frac{M(1-\beta+\eta) t}{(1-\beta)(1+\alpha+\eta)} I_{0, t}^{\alpha, \beta, \eta}\{f(t)\}+M I_{0, t}^{\alpha, \beta, \eta}\{t f(t)\},
$$

for all $t>0, \alpha>0, \beta<1, \beta-1<\eta<0$.

Indeed, by suitably specializing the values of parameters $\mu$ and $\beta$ the results presented in this paper would find further fractional inequalities involving the Erdélyi-Kober and Riemann-Liouville type fractional integral operator, on taking relations (26) and (27) into account. For example, if we set $\mu=0$ and $\beta=0$, Theorems 10 and 11 lead to the following results involving Erdélyi-Kober fractional integral operator.

Corollary 14. Let $f$ and $g$ be two functions defined on $[0, \infty)$, such that $f$ is increasing, $g$ is differentiable, and there exists a real number $m=\inf _{t \geq 0} g^{\prime}(t)$; then

$$
\begin{aligned}
& I^{\alpha, \eta}\{f(t) g(t)\} \\
& \geq \frac{\Gamma(1+\alpha+\eta)}{\Gamma(1+\eta)} I^{\alpha, \eta}\{f(t)\} I^{\alpha, \eta}\{g(t)\} \\
& \quad-\frac{m(1+\eta) t}{(1+\alpha+\eta)} I^{\alpha, \eta}\{f(t)\}+m I^{\alpha, \eta}\{t f(t)\},
\end{aligned}
$$

for all $t>0, \alpha>0,-1<\eta<0$.

Corollary 15. Let $f$ and $g$ be two functions defined on $[0, \infty)$, such that $f$ is increasing, $g$ is differentiable and there exists a real number $M=\sup _{t \geq 0} g^{\prime}(t)$, then

$$
\begin{aligned}
& I^{\alpha, \eta}\{f(t) g(t)\} \\
& \geq \frac{\Gamma(1+\alpha+\eta)}{\Gamma(1+\eta)} I^{\alpha, \eta}\{f(t)\} I^{\alpha, \eta}\{g(t)\} \\
& \quad-\frac{M(1+\eta) t}{(1+\alpha+\eta)} I^{\alpha, \eta}\{f(t)\}+M I^{\alpha, \eta}\{t f(t)\},
\end{aligned}
$$

for all $t>0, \alpha>0,-1<\eta<0$.

Finally, if we take $\mu=0$ and $\beta=-\alpha(\nu=0$ and $\delta=-\gamma$ additionally for Theorem 6), then Theorems 5 to 11, yield the known result due to Belarbi and Dahmani [2].

We conclude with the remark that the results derived in this paper are general in character and give some contributions to the theory integral inequalities and fractional calculus. Moreover, they are expected to find some applications for establishing uniqueness of solutions in fractional boundary value problems and in fractional partial differential equations.

\section{Conflict of Interests}

The authors declare that there is no conflict of interests regarding the publication of this paper.

\section{References}

[1] G. A. Anastassiou, Advances on Fractional Inequalities, Springer Briefs in Mathematics, Springer, New York, NY, USA, 2011.

[2] S. Belarbi and Z. Dahmani, "On some new fractional integral inequalities," Journal of Inequalities in Pure and Applied Mathematics, vol. 10, no. 3, article 86, 5 pages, 2009.

[3] Z. Dahmani, O. Mechouar, and S. Brahami, "Certain inequalities related to the Chebyshev's functional involving a type Riemann-Liouville operator," Bulletin of Mathematical Analysis and Applications, vol. 3, no. 4, pp. 38-44, 2011.

[4] Z. Denton and A. S. Vatsala, "Monotonic iterative technique for finit system of nonlinear Riemann-Liouville fractional differential equations," Opuscula Mathematica, vol. 31, no. 3, pp. 327-339, 2011.

[5] S. S. Dragomir, "Some integral inequalities of Grüss type," Indian Journal of Pure and Applied Mathematics, vol. 31, no. 4, pp. 397-415, 2000.

[6] S. L. Kalla and A. Rao, "On Grüss type inequality for hypergeometric fractional integrals," Le Matematiche, vol. 66, no. 1, pp. 57-64, 2011.

[7] V. Lakshmikantham and A. S. Vatsala, "Theory of fractional differential inequalities and applications," Communications in Applied Analysis, vol. 11, no. 3-4, pp. 395-402, 2007.

[8] H. Öğünmez and U. M. Özkan, "Fractional quantum integral inequalities," Journal of Inequalities and Applications, vol. 2011, Article ID 787939, 7 pages, 2011.

[9] S. D. Purohit and R. K. Raina, "Chebyshev type inequalities for the saigo fractional integrals and their q-analogues," Journal of Mathematical Inequalities, vol. 7, no. 2, pp. 239-249, 2013.

[10] J. D. Ramírez and A. S. Vatsala, "Monotonic iterative technique for fractional differential equations with periodic boundary conditions," Opuscula Mathematica, vol. 29, no. 3, pp. 289-304, 2009.

[11] W. T. Sulaiman, "Some new fractional integral inequalities," Journal of Mathematical Analysis, vol. 2, no. 2, pp. 23-28, 2011.

[12] P. L. Chebyshev, "Sur les expressions approximatives des integrales definies par les autres prises entre les mêmes limites," Proceedings of the Mathematical Society of Kharkov, vol. 2, pp. 93-98, 1882.

[13] L. Curiel and L. Galué, "A generalization of the integral operators involving the Gauss' hypergeometric function," Revista Técnica de la Facultad de Ingeniería Universidad del Zulia, vol. 19, no. 1, pp. 17-22, 1996.

[14] V. S. Kiryakova, Generalized Fractional Calculus and Applications, Pitman Research Notes in Mathematics Series no. 301, Longman Scientific \& Technical, Harlow, UK, 1994.

[15] A. M. Mathai, R. K. Saxena, and H. J. Haubold, The H-Function: Theory and Applications, Springer, Dordrecht, The Netherlands, 2010.

[16] M. Saigo, "A remark on integral operators involving the Gauss hypergeometric functions," Mathematical Reports, Kyushu University, vol. 11, pp. 135-143, 1978. 


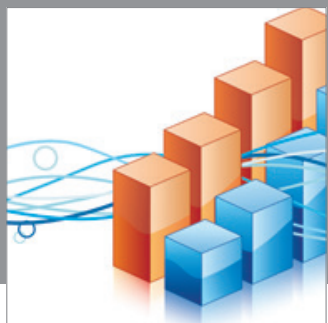

Advances in

Operations Research

mansans

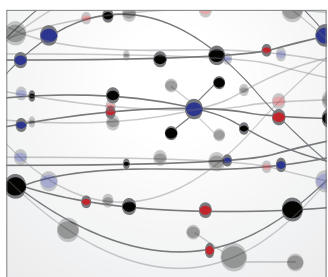

The Scientific World Journal
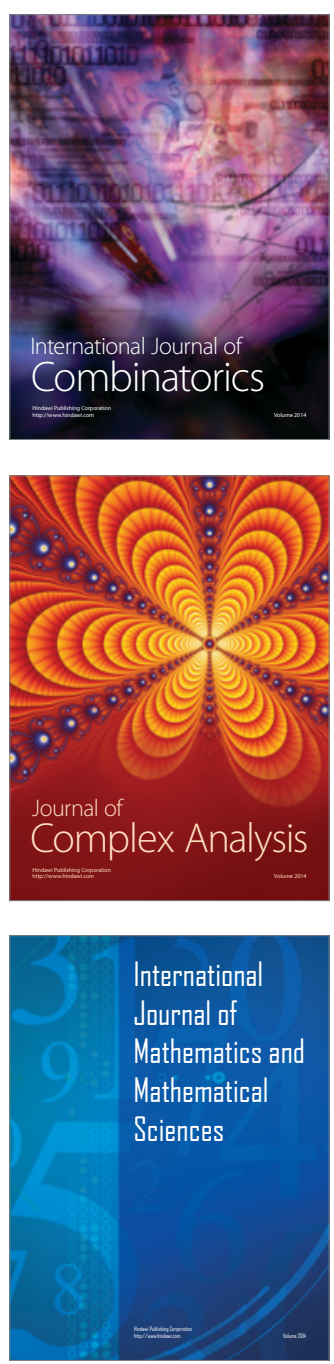
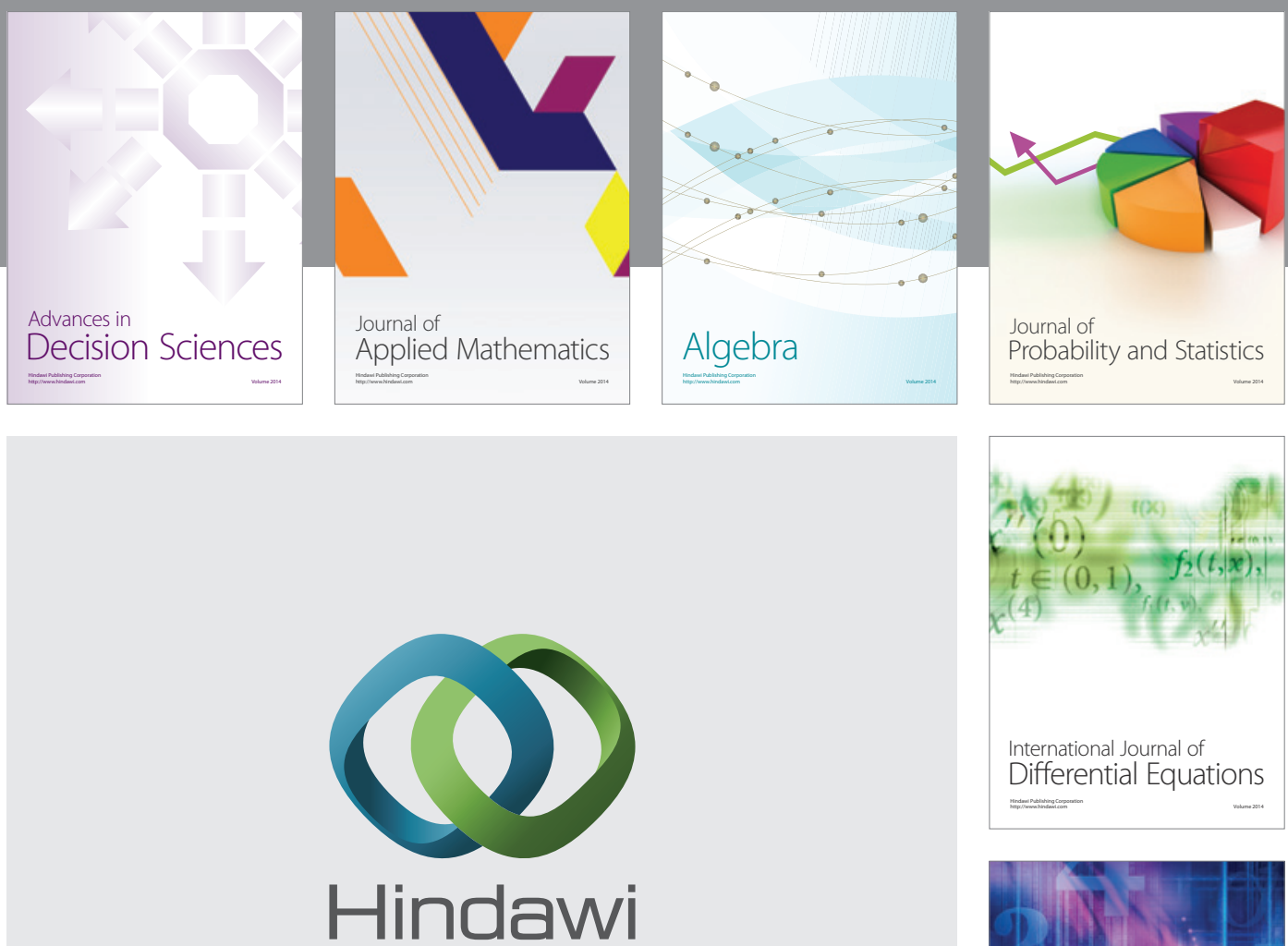

Submit your manuscripts at http://www.hindawi.com
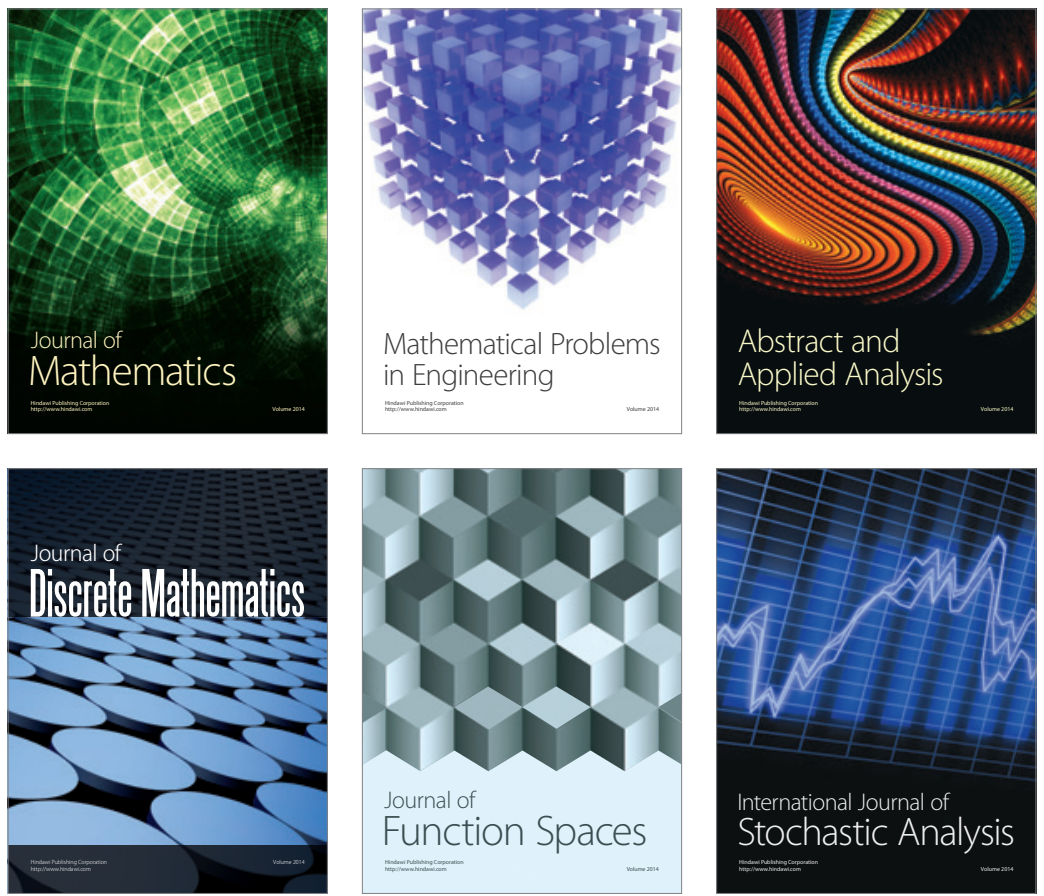

Journal of

Function Spaces

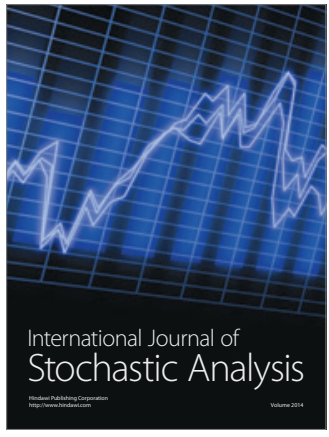

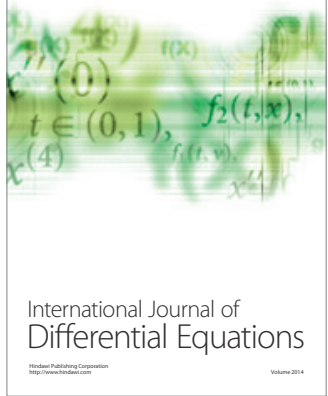
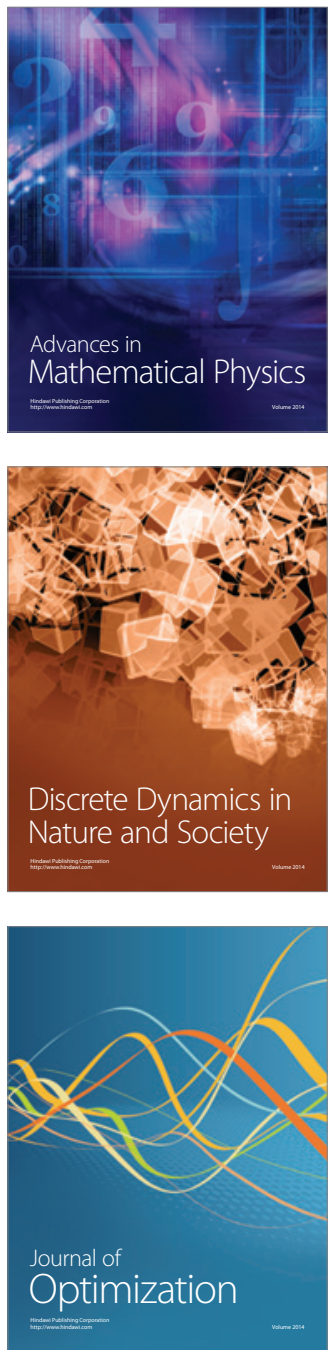